\title{
Liquid atmospheric pressure matrix- assisted laser desorption/ionization mass spectrometry adds enhanced functionalities to MALDI MS profiling for disease diagnostics
}

Article

Published Version

Creative Commons: Attribution 4.0 (CC-BY)

Open Access

Hale, O. J., Morris, M., Jones, B., Reynolds, C. K. and Cramer, R. (2019) Liquid atmospheric pressure matrix-assisted laser desorption/ionization mass spectrometry adds enhanced functionalities to MALDI MS profiling for disease diagnostics. ACS Omega, 4 (7). pp. 12759-12765. ISSN 2470-1343 doi: https://doi.org/10.1021/acsomega.9b01476 Available at https://centaur.reading.ac.uk/85319/

It is advisable to refer to the publisher's version if you intend to cite from the work. See Guidance on citing.

To link to this article DOI: http://dx.doi.org/10.1021/acsomega.9b01476

Publisher: ACS

All outputs in CentAUR are protected by Intellectual Property Rights law, including copyright law. Copyright and IPR is retained by the creators or other copyright holders. Terms and conditions for use of this material are defined in the End User Agreement. 


\section{www.reading.ac.uk/centaur}

\section{CentAUR}

Central Archive at the University of Reading

Reading's research outputs online 


\title{
Liquid Atmospheric Pressure Matrix-Assisted Laser Desorption/ Ionization Mass Spectrometry Adds Enhanced Functionalities to MALDI MS Profiling for Disease Diagnostics
}

\author{
Oliver J. Hale, ${ }^{\dagger, \|}$ Michael Morris, ${ }^{\ddagger}$ Barney Jones, ${ }^{\S}$ Christopher K. Reynolds, ${ }^{\S}$ and Rainer Cramer* ${ }^{\dagger \dagger}$ \\ ${ }^{\dagger}$ Department of Chemistry, University of Reading, Whiteknights, Reading RG6 6AD, U.K. \\ ${ }^{t}$ Waters Corporation, Stamford Avenue, Wilmslow SK9 4AX, U.K. \\ ${ }^{\S}$ The Centre for Dairy Research, School of Agriculture, Policy and Development, University of Reading, Reading RG2 9HX, U.K.
}

Supporting Information

ABSTRACT: A liquid matrix-assisted laser desorption/ionization (liquid MALDI) method has been developed for high-throughput atmospheric pressure (AP) mass spectrometry (MS) analysis of the molecular content of crude bioliquids for disease diagnostics. The presented method is rapid and highly robust, enabling its application in environments where speed and low-cost high-throughput analyses are required. Importantly, because of the creation of multiply charged analyte ions, it provides

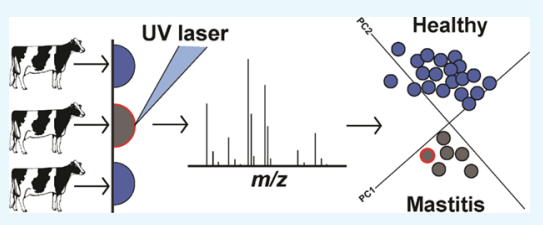
additional functionalities that conventional solid MALDI MS profiling is lacking, including the use of high-performance mass analyzers with limited $\mathrm{m} / z$ range. The concomitant superior MS/MS performance that is achieved similar to ESI MS/MS adds greater analytical power and specificity to MALDI MS profiling while retaining the advantages of a fast laser-based analysis system and off-line large-scale sample preparation. The potential of this MALDI MS profiling method is demonstrated on the detection of dairy cow mastitis, which is a substantial economic burden on the dairy industry with losses of hundreds of dollars per diseased cow per year, equating to a total annual loss of billions of dollars, as well as leading to the use of large quantities of antibiotics, adding to the proliferation of antimicrobial resistance. Only small amounts of aliquots obtained from the daily farm milking process were prepared for liquid MALDI MS profiling using a simple one-pot/two-step analyte extraction. Automated analysis was performed using a custom-built AP-MALDI ion source, enabling the simultaneous detection of lipids, peptides, and proteins. Diagnostic, multiply charged, proteinaceous ions were easily sequenced and identified by MS/MS experiments. Samples were classified according to mastitis status using multivariate analysis, achieving $98.5 \%$ accuracy (100\% specificity) determined by "leave $20 \%$ out" cross-validation. The methodology is generally applicable to AP-MALDI MS profiling on most commercial high-resolution mass spectrometers, with the potential for expansion into hospitals for rapid assessment of human and other biofluids.

\section{INTRODUCTION}

Conventional (solid) matrix-assisted laser desorption/ionization mass spectrometry (MALDI MS) profiling has been applied in many areas of disease analysis with varying success. In general, blood analysis by MALDI MS profiling (and its various incarnations such as SELDI ${ }^{1}$ ) has had limited success because of many reasons, including the limited analytical power of the typically used axial time-of-flight (TOF) mass analyzers with respect to MS/MS analysis and the mass resolution and accuracy that is ultimately achievable. However, MALDI MS profiling in clinical microbiology is one of the success stories of MALDI, ${ }^{2}$ although the same limitations with regard to ionization and instrumentation remain.

Recently, liquid atmospheric pressure (AP)-MALDI MS was introduced as an alternative to conventional solid MALDI with many of the key advantages of electrospray ionization (ESI) such as the generation of multiply charged ions and thus the option to use high-performance (hybrid) mass analyzers such as QTOFs and Orbitraps that require analyte ions with low $\mathrm{m} /$ $z$ values. ${ }^{3}$ While conventional MALDI predominately produces singly charged ions, liquid MALDI produces "ESI-like" multiply charged ions, resulting in low $m / z$ values. In addition, multiply charged ions typically lead to superior fragmentation in collision-induced dissociation (CID)-based MS/MS (cf. peptide sequencing) and are a requirement for electronmediated MS/MS analysis. ${ }^{4,5}$ Ultraviolet photodissociation improves the fragmentation of singly charged protein ions, but the technology has limited availability on commercial instrumentation, and the requirement of high $\mathrm{m} / \mathrm{z}$ mass analyzers remains for singly charged protein ions. ${ }^{4}$ Advantageously, liquid MALDI also benefits from virtually all of the advantages of solid MALDI, such as high tolerance toward sample additives/contaminants, post-analysis sample recovery and storage, and all of the advantages of a laser-based method, such as speed and a tightly controllable desorption event, while adding additional key features such as greater sample homogeneity, greater ion beam stability, and lower sample consumption. ${ }^{6}$

Received: $\quad$ May 21, 2019

Accepted: July 15, 2019

Published: July 26, 2019 
The combination of all of these additional features and the well-known strengths of conventional MALDI makes liquid MALDI a prime candidate for the development of a powerful MS profiling method that can now add the advances made in modern ESI MS to the speed, robustness, and easiness of MALDI MS analysis. Following this rationale, the recently introduced liquid AP-MALDI MS profiling methodology ${ }^{3}$ was adapted for the analysis of crude bioliquids. As a proof-ofconcept application, milk directly obtained from daily farm milking was analyzed for the accurate detection of mastitis.

Mastitis, an infection of the mammary gland, is one of the most prevalent diseases in dairy cattle and a significant cause of economic loss in the dairy industry; yet, early diagnosis is still a considerable challenge. $^{7-9}$ The molecular composition of milk is subject to variation with the animal's health. ${ }^{10,11}$ Milk is thus of interest as a source of information for disease diagnosis that is collected daily and an alternative to other body fluids such as blood, saliva, and urine, which are more intrusive or difficult to collect routinely on a farm.

Current milk analysis methods, such as the California mastitis test (CMT), ${ }^{12}$ produce results largely open to interpretation by the operator and have debatable sensitivity and specificity, while rigorous bacteriological assessments take days to perform. ${ }^{13-16}$ Flow cytometry is routinely used to measure somatic cell concentration in milk samples, which is often increased in response to mastitis, but somatic cell count is determined by numerous factors in addition to immune response to infection. ${ }^{17}$

Validated milk molecular biomarkers for diagnosing mastitis have yet to be described, ${ }^{9}$ but several candidates have been proposed. ${ }^{18-23}$ Once validated molecular biomarkers are available, targeted assays for their specific detection and quantitation, such as immunoassays, are typically developed. Disappointingly, these classical diagnostic tests often reach their limits with respect to marker specificity and multiplexing, to name but a few.

An alternative approach is MS profiling using well-defined classification models from multivariate analysis, exploiting the richness of the samples' biomolecular profiles detected by highresolution MS. Multivariate analysis has been used to great effect utilizing MS profiles produced with electrosurgical tools (e.g., "iKnife") and laser desorption probes to rapidly distinguish between healthy and malignant tissue. ${ }^{24,25}$ However, these techniques have focused on analyzing small, abundant molecules such as phospholipids, limiting access to biomarkers that may exist as proteinaceous molecules. ${ }^{24,25}$ Results are typically determined by computational modeling, which reduces the potential of operator-related bias and produces a recorded, reviewable raw data trail. Similarly, MS profiling has been introduced to clinical microbiology with great success, reducing the time to diagnosis by many hours (and often days) with high identification accuracy. ${ }^{26}$ The latter application of MS profiling is achieved using MALDI in its conventional form and implementation, that is, preparing solidstate MALDI samples that are analyzed in an axial TOF instrument under high vacuum. Unfortunately, classical MALDI MS analysis is limited with respect to sample homogeneity and thus ion beam stability (cf. hunting for the so-called "sweet spot"), speed of sample preparation, introduction, and analysis as well as subsequent identification/verification of the main diagnostic contributors in the recorded MS profile (i.e., poor structurally informative MS/MS performance).
Here, we present a method for rapidly analyzing and classifying milk samples by AP-MALDI MS using 100\% liquid MALDI samples and their associated advantages. The potential of "on-the-fly" MS/MS analysis of multiply charged analyte ions for greater analytical specificity, as well as the use of modern bioanalytics developed for clinical applications, significantly adds to the power of this MS method and is demonstrated in this study.

\section{RESULTS AND DISCUSSION}

Raw milk samples were collected from individual cows at the Centre for Dairy Research at the University of Reading, UK, and prepared for liquid MALDI MS analysis by a one-pot/twostep extraction, taking less than $5 \mathrm{~min}$. Samples were taken from individual udder quarters of 109 cows for a total of 135 samples, with some cows being sampled on multiple dates. Representative mass spectra of the milk extracts from a cow from each of the two investigated classes (mastitis and healthy-see the Experimental Section for further details) are shown in Figure S1. Phospholipids were abundant in the $\mathrm{m} / z$ range of $600-1000$. In addition, many multiply charged ions attributable to peptides were detected in this $m / z$ range and beyond, with high abundance in the mastitis samples (Figure S2). Deconvolution of the spectra suggests that multiply charged ions above $m / z 600$ are attributable to proteins (e.g., $\beta$-casein) or larger protein fragments (Table 1, Figure 1).

Table 1. Deconvoluted Highly Charged Liquid AP-MALDI MS Ions and Their Class Association ${ }^{a}$

$\begin{array}{cccc}\begin{array}{c}\text { liquid AP-MALDI MS profile ions } \\ \text { (most intense } m / z)\end{array} & \begin{array}{c}\text { deconvoluted } \\ \text { mass }(\mathrm{Da})\end{array} & \text { healthy } & \text { mastitis } \\ 477.28^{4+}, 636.37^{3+}, 954.55^{2+} & 1905 & & \times \\ 583.34^{5+}, 728.67^{4+}, 971.56^{3+} & 2910 & \times \\ 664.87^{6+}, 797.45^{5+} & 3981 & \times & \times \\ 620.94^{7+}, 724.25^{6+}, 868.90^{5+}, & 4340 & & \times \\ 1085.88^{4+}, 1447.82^{3+} & & & \times \\ 644.39^{8+}, 736.15^{7+} & 5145 & \times \\ 1046.82^{5+}, 1307.75^{4+} & 5226 & \\ 1224.30^{7+}, 1428.32^{6+} & 8561 & & \times \\ 1051.01^{9+}, 1182.17^{8+}, 1351.02^{7+}, & 9450 & & \times \\ 1576.37^{6+} & 10551 & & \times \\ 812.78^{13+}, 880.52^{12+}, 960.55^{11+} & 11820 & \times & \times \\ 1183.13^{10+}, 1314.81^{9+}, 1478.88^{8+}, & 18360 & \times & \times \\ 1689.94^{7+} & 24020 & \times & \\ 1413.74^{13+}, 1531.12^{12+}, 1670.31^{11+} & & \end{array}$

${ }^{a}$ Crosses $(\times)$ indicate that signals for these ions were detected in MS profile spectra for the specified class and contributed to the classification of the samples as such in multivariate analysis.

For sample classification, a multivariate model featuring 10 principal component analysis (PCA) dimensions and 1 linear discriminant analysis (LDA) dimension was constructed from a pool of 73 "healthy" and 62 "mastitis" TOF MS profiles acquired in an automated sequence from randomly spotted MALDI samples (example chromatogram in Figure S3). Analysis was performed with the Abstract Model Builder (AMX [Beta] version 1.0.1563.0) software developed by Waters. The projection of the first two PCA dimensions is shown in Figure 2a, with the second dimension offering visual distinction between the two classes. The loading plot is displayed in Figure 2b, which shows a multitude of 
Healthy
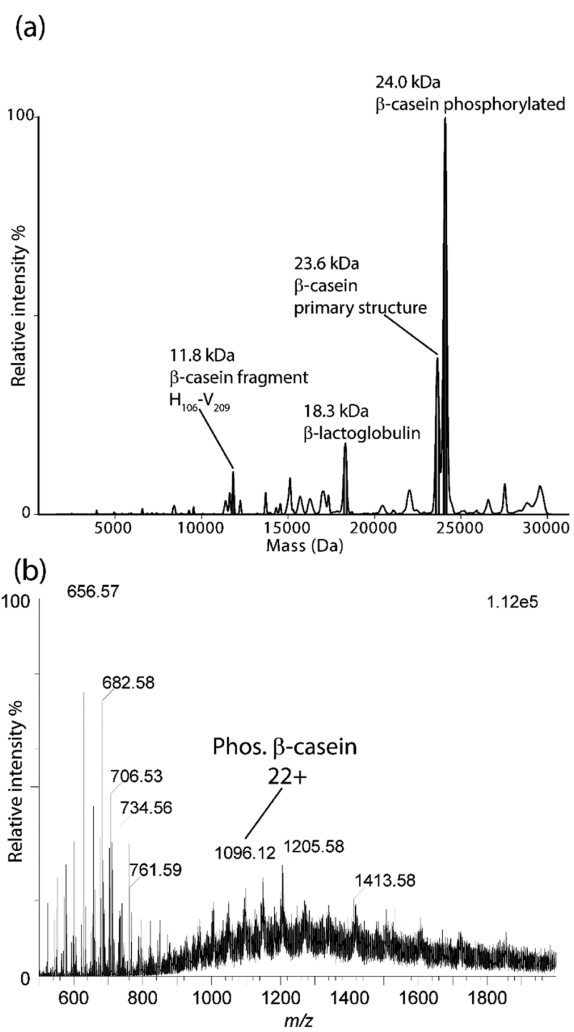

Mastitis

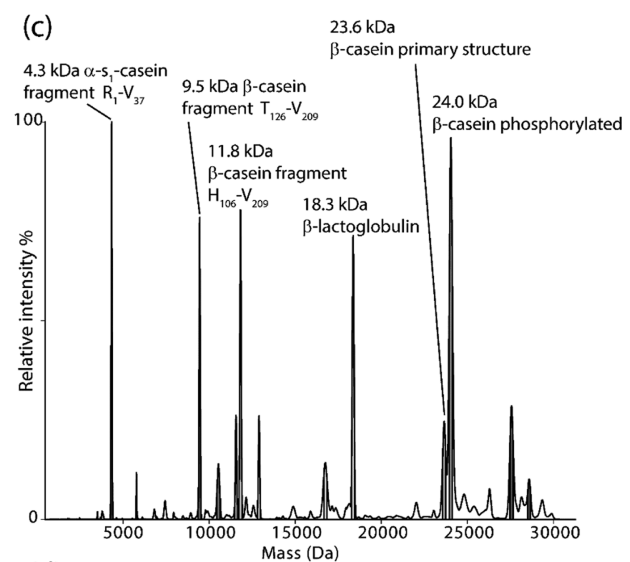

(d) $6+\ldots-\alpha-5-$ cassein

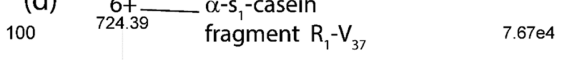

$\beta$-casein fragment

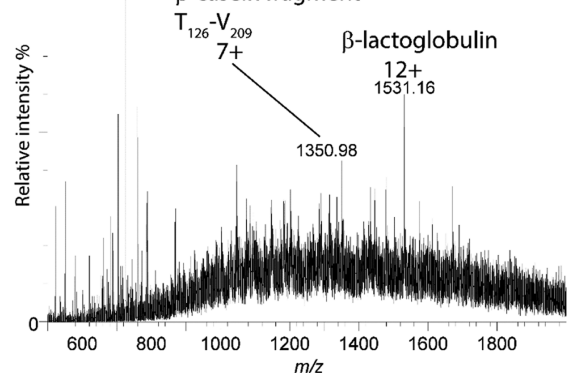

Figure 1. Examples of deconvoluted mass spectra $(a, c)$ obtained from spectra showing multiply charged ion species detected from representative "healthy" and "mastitis" milk samples (b,d). Molecules as large as $\beta$-casein were detected as multiply charged ions. Lower mass components with strong ion signal intensities are also evident in the deconvoluted mastitis spectra, indicating protein degradation. Some diagnostic ions and their charge states are indicated in the raw mass spectra assigned by deconvoluted mass and CID MS/MS experiments discussed later in this article. Absolute detector ion counts for $100 \%$ signal intensity can be found in the top right corner of the nondeconvoluted mass spectra (b,d).

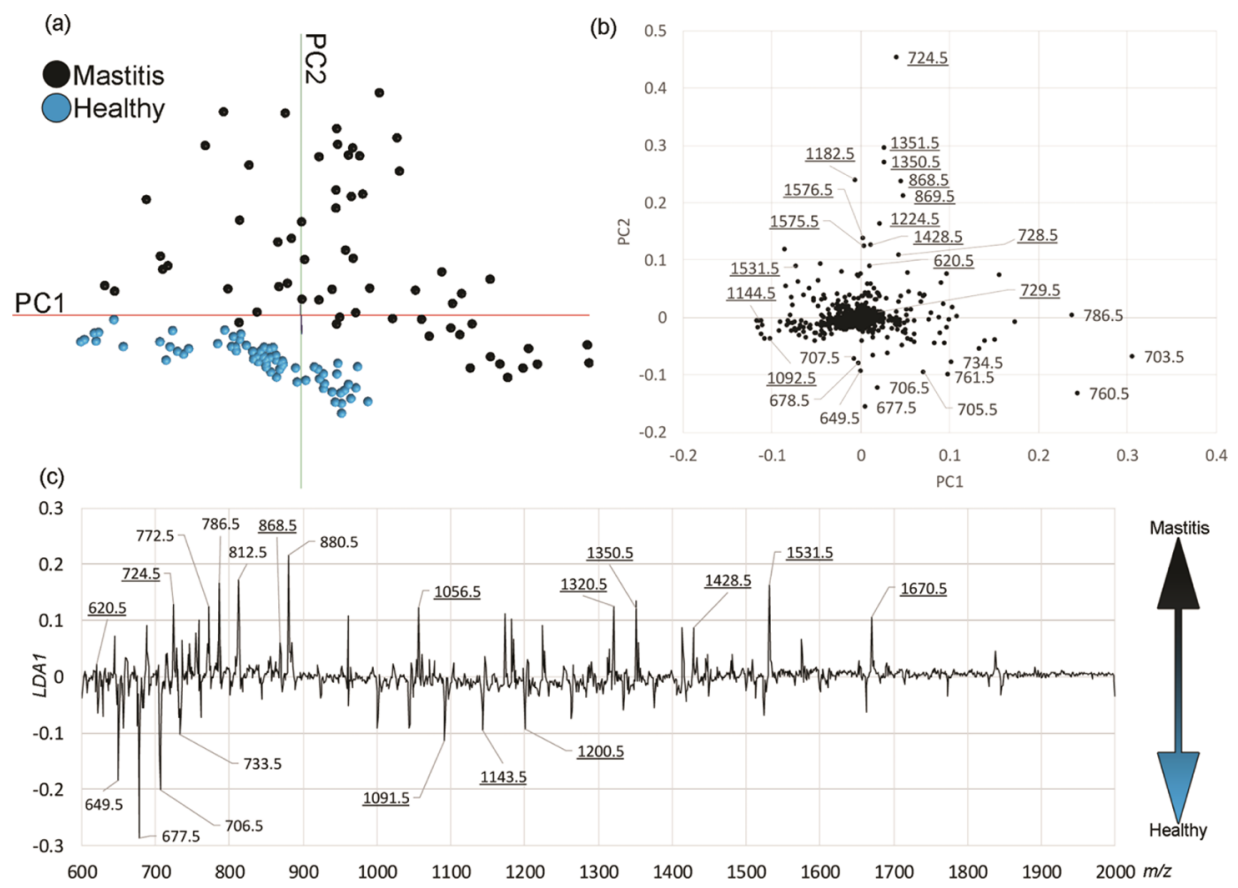

Figure 2. Classification of milk samples from "healthy" and "mastitis" cows by liquid AP-MALDI MS and multivariate model building. (a) Plot of the first two PCA dimensions, showing separation of the two classes. (b) Loading plot for dimensions 1 and 2 reveals the most influential ion peaks for the PCA classification in (a). Ions with underlined labels have charge states greater than 1. (c) Mass loading plot for the LDA model generated from the PCA model. Peaks of greatest positive/negative intensity are the most influential for classification. 


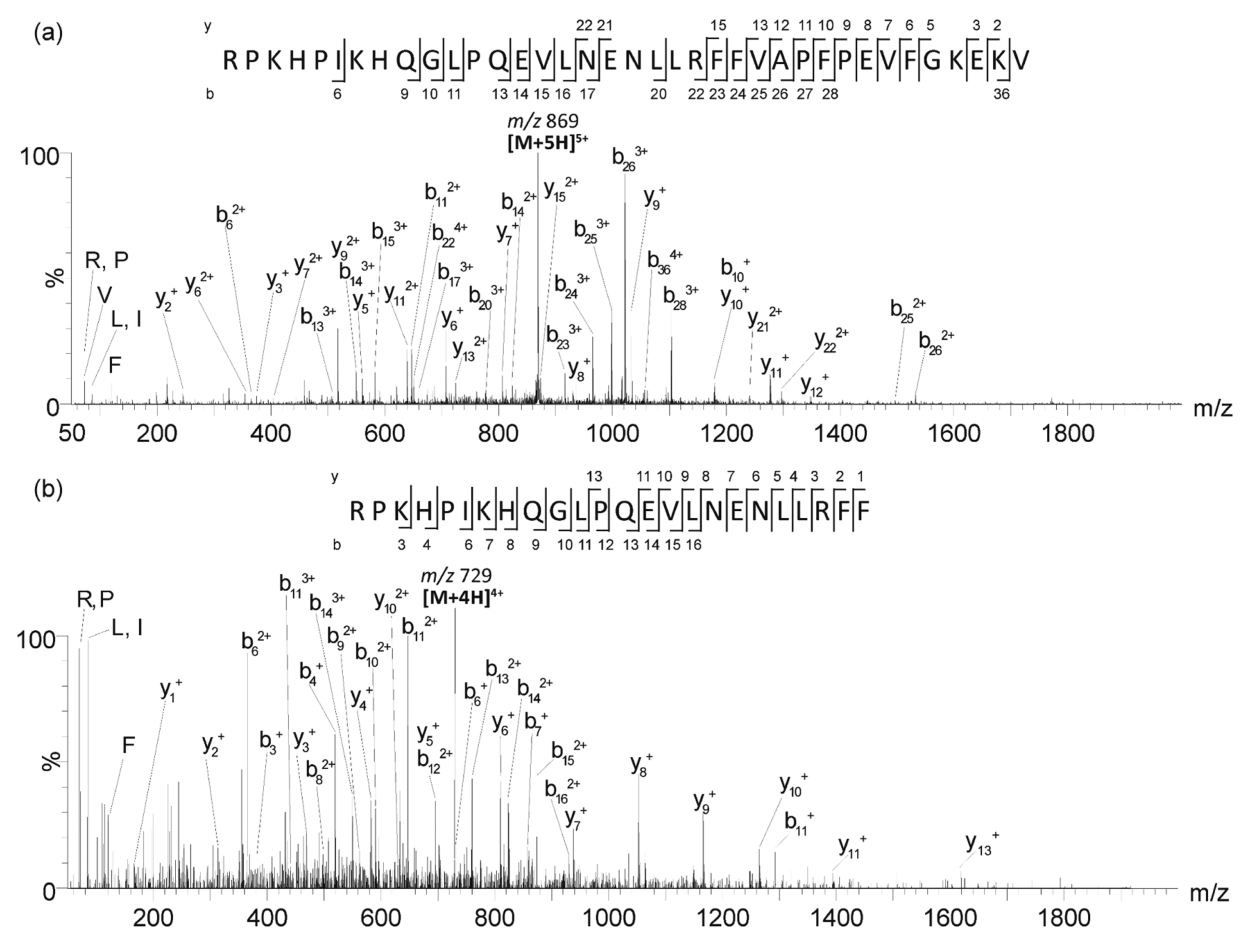

Figure 3. Liquid AP-MALDI-CID MS/MS spectra of MS profile ion peaks. (a) MS/MS spectrum of the diagnostically important precursor ion [M $+5 \mathrm{H}]^{5+}$ (approx. $m / z$ 869) and (b) MS/MS spectrum of the diagnostically less important $[\mathrm{M}+4 \mathrm{H}]^{4+}$ (approx. $m / z$ 729); both spectra are annotated to indicate $y, b$, and immonium ions. The proposed sequences are displayed above the spectrum and indicate $\mathrm{N}$-terminal fragments $\left(\mathrm{R}_{1}\right.$ $\mathrm{V}_{37}$ and $\mathrm{R}_{1}-\mathrm{F}_{24}$, respectively) derived from $\alpha$-s $\mathrm{s}_{1}$-casein (P02662).

phospholipid, peptide, and protein ions as being influential on the second dimension. The LDA mass loading plot (Figure 2c) shows the influence of ions in the classification model. The model's classification performance was assessed by the built-in "leave 20\% out" cross-validation method of AMX. From this cross-validation, a sensitivity of $90 \%$ (56/62 mastitis spectra) and a specificity of $100 \%$ (73/73 healthy spectra) are obtained with a total classification accuracy of $95.56 \%$, demonstrating the potential for this method as a routine test. Importantly, no false positives were reported.

In principle, the detected proteinaceous diagnostic ions are of interest as milk composition changes can often be attributed to disease-related action of endogenous (and/or exogenous) enzymes. While current MALDI MS profiling methods fall short of providing enhanced capabilities in identifying diagnostic peptides or proteins because of poor MS/MS performance, the predominant production of multiply charged precursor ions in liquid AP-MALDI MS profiles now enables the employment of "ESI-like" MS/MS sequencing, using highperformance MS/MS instruments with a small $\mathrm{m} / \mathrm{z}$ range such as hybrid Orbitrap and QTOF mass spectrometers. Thus, to provide molecular structural information, some multiply charged ions were selected for AP-MALDI CID MS/MS using the same liquid MALDI sample from which the MS profile was acquired. This "top-down" approach required no further sample preparation. Generally, MS/MS spectra were acquired in less than $5 \mathrm{~min}$ (dependent on precursor ion intensity) and typically provide greater diagnostic information if acquired from multiply charged ions compared to singly charged ions. $^{3,27}$

The presence of the precursor ion at approx. $m / z 869$ (Figure 3a) contributed strongly to the classification of samples as "mastitis", whereas the ion at approx. $m / z 729$
(Figure 3b) was not strongly influential for either class. The identity of the ion at $m / z 869$ can be assigned to the amino acid sequence $\mathrm{R}_{1}-\mathrm{V}_{37}$ of $\alpha$-s $\mathrm{s}_{1}$-casein (Uniprot P02662), an abundant milk protein. This peptide has been reported by other studies but has not been attributed to the action of a known endogenous (or exogenous) enzyme. ${ }^{20,28,29}$ The identity of this unknown enzyme could be important for targeted mastitis detection. The second ion at $m / z 729$ can be derived from the proteolysis of $\alpha$-s s $_{1}$ casein by cathepsin B or D, producing a peptide composed of amino acids $\mathrm{R}_{1}-\mathrm{F}_{24}{ }^{29}$ However, because this peptide does not strongly influence the mastitis classification, the action of cathepsins B and D is less likely to be related to mastitis infection. Both peptides contain the sequence of a known antimicrobial peptide (isracidin, $\alpha$-s s $^{-}$ casein amino acids $1-23) .^{30}$

Furthermore, plasmin-related activity is suggested to result in two $\beta$-casein-derived peptides identified in Figure S4, the smaller of which was detected exclusively in the mastitis samples and contributed strongly to their classification as such. Increased enzymatic activity, such as plasmin proteolysis, has long been implicated as a factor in mastitis diagnosis. ${ }^{31}$

Using this information, a targeted PCA/LDA model was built from the same data as the original model over a narrow $m / z$ range $(m / z 720-m / z 740)$ that includes three peptide ions at approximately $m / z 724, m / z 728$, and $m / z 736$ (see Table 1). This resulted in an increase in classification accuracy to $98.52 \%$ when assessed by "leave $20 \%$ out" cross-validation, while maintaining a specificity of $100 \%$. Only two mastitis samples were misclassified. Interestingly, milking record data showed that these two samples were collected after an initial mastitis treatment with antibiotics 1 week prior to sampling for the present study. It is possible that these animals were 
recovering and thus expressed the markers of mastitis more weakly.

\section{CONCLUSIONS}

Besides the method's high classification accuracy, the generation of "ESI-like" multiply charged precursor ions facilitates the MS/MS identification and characterization of diagnostically important profile ions beyond what is currently possible with MALDI MS profiling methods. Consequently, the improved MALDI MS/MS capabilities of the presented method enables the collection of further information about the underlying disease biology and for the potential development of well-defined targeted assays. However, there is also great potential for multiplexing in disease detection using liquid APMALDI MS profiling because of the acquisition of an intrinsically rich biomolecular profile, thus providing a more comprehensive health check from a single sample analysis. Importantly, the method is easily automated and capable of large-scale analyses as needed for high-throughput testing of millions of milk samples, which is the current yearly level of milk testing in the UK's dairy industry alone (https://www. nmr.co.uk/). We anticipate that planned automation and ion source improvements will further decrease analysis time and that additional diagnostic applications for other crude bioliquids will be reported in the future. This could include the assessment of human milk samples for mastitis in hospitals, where MS is already used in many areas, from screening newborn babies for disorders to clinical microbiology where conventional solid MALDI MS has already shown its diagnostic advantages.

\section{EXPERIMENTAL SECTION}

Materials. Water, acetonitrile $(\mathrm{MeCN})$, isopropanol (IPA), and trifluoroacetic acid (all HPLC-grade) were bought from Fisher Scientific (Loughborough, UK). Sodium iodide solution was purchased from Waters (Manchester, UK). All other matrix components, reagents, calibrants, and analytical standards were acquired from Sigma-Aldrich (Gillingham, UK).

Cow Milk Samples. Raw milk samples were collected from individual cows from February 2016 to December 2017 at the Centre for Dairy Research, a facility at the University of Reading (https://www.reading.ac.uk/apd/research-andfacilities/cedar.aspx). Samples were taken from one udder quarter per cow on a given day. Cows were assessed at each milking (twice daily) by fore-milking each udder teat prior to attachment of the milking apparatus. If clots were present from a quarter, the cow was determined to have clinical mastitis and milk was collected from the affected teat. Mastitis samples were immediately obtained from the affected udder quarter by handmilking and frozen at $-80^{\circ} \mathrm{C}$. Milk samples for analysis ranged in appearance from obvious clinical infections (yellowed, clotted) to indistinguishable from healthy samples to the untrained eye. Subsequent confirmation of infection was obtained by physical symptoms (body temperature and/or mammary swelling) and analysis of milk for somatic cell count or culture to identify the infecting microorganism. Bacterial culture and assessment by MALDI-TOF MS profiling were performed by Quality Milk Management Services Ltd. (QMMS, Easton, UK).

Analyte Extraction. Aqueous trichloroacetic acid solution $(250 \mu \mathrm{L}, 5 \% \mathrm{~m} / \mathrm{v})$ was added to raw milk samples $(50 \mu \mathrm{L})$ and briefly vortexed. Following room-temperature centrifugation at
$13000 \mathrm{rpm}$ for $2 \mathrm{~min}$, the supernatant was discarded, and the precipitate was resolubilized in sodium hydroxide $(200 \mu \mathrm{L}, 0.5$ $\mathrm{mM}$ in 1:1 $\mathrm{H}_{2} \mathrm{O} / \mathrm{IPA}$ ) with ultrasonic assistance. This simple extraction removed a significant amount of lactose and related polysaccharides, which otherwise dominate the mass spectrum. These are evident in other published work featuring only a water/methanol dilution. ${ }^{32}$

Liquid MALDI Sample Preparation. The liquid support matrix (LSM) consisted of 2,5-DHB solution $(25 \mathrm{mg} / \mathrm{mL}, 3: 7$ $\mathrm{H}_{2} \mathrm{O} / \mathrm{MeCN}$ ) with ethylene glycol added equal to $60 \%$ of the solution volume. This modification to our usual glycerol-based LSM stemmed from recent work optimizing ion signal through substitution of glycerol with other diols. ${ }^{33}$

Per sample, LSM (750 nL) was deposited onto a Waters stainless steel MALDI target. Analyte solution $(750 \mathrm{~nL})$ was subsequently added to the LSM droplets. Typical droplet shape was that of a dome and was considered optimal for robust ion signal. Samples were analyzed immediately after spotting without any additional drying time.

AP-MALDI MS Data Acquisition. MS analysis was performed with a Synapt G2-Si HDMS mass spectrometer (Waters Corporation, Wilmslow, UK) equipped with an inhouse developed AP-MALDI source, featuring a Waters Research Enabled Software (WREnS)-controlled XY-stage (Zaber Technologies Inc., Vancouver, Canada). This setup is capable of generating multiply charged ions and has been detailed previously. ${ }^{34}$ In brief, an MNL100 nitrogen laser (LTB Lasertechnik Berlin, Berlin, Germany) with a wavelength of $337 \mathrm{~nm}$, a maximum pulse repetition rate of $30 \mathrm{~Hz}$, and a pulse width of approximately 3 ns was attenuated by a neutral density filter to $20-30 \mu \mathrm{J} /$ pulse for AP-MALDI MS measurements. A stainless-steel ion transfer tube $(60 \mathrm{~mm}$ length, $1 \mathrm{~mm}$ internal diameter) was added as the first ion extracting element to the ion block with a gap of approximately $3 \mathrm{~mm}$ between the MALDI target plate and inlet of the ion transfer tube. Approximately $26 \mathrm{~W}$ was delivered by a low-voltage DC power supply to a wire (approx. $6 \Omega$ resistance) surrounding the transfer tube, providing heating. In contrast to other experiments conducted with this source, no counter-flow $\mathrm{N}_{2}$ gas was supplied to aid desolvation as it was observed to increase alkali metal adduct formation for the highly charged ions. This resulted in an internal transfer tube temperature of approx. $100{ }^{\circ} \mathrm{C} .{ }^{34}$ MALDI target voltage was set to $3-4 \mathrm{kV}$ and the cone voltage to $30-40 \mathrm{~V}$. The instrument was operated in ion mobility-TOF mode, enabling ion mobility measurements to be recorded alongside $\mathrm{m} / z$.

With the $337 \mathrm{~nm}$ laser firing at $30 \mathrm{~Hz}$, data were acquired for $60 \mathrm{~s}$ per sample droplet at a rate of 1 TOF scan per second. The mass spectrometer was calibrated over the $m / z$ range $100-2000$ with a mixture of polyethylene glycol $(0.1 \mathrm{mg} / \mathrm{mL}$; $M_{\mathrm{w}} 200,600$, and 2000$)$ or sodium iodide $(2 \mu \mathrm{g} / \mu \mathrm{L}$ in $50 \%$ IPA). Lock mass correction was applied using the peak of PC (16:0/18:1) $(\mathrm{m} / z$ 760.5851), an abundant phosphatidylcholine in bovine milk. CID MS/MS experiments were performed for up to $5 \mathrm{~min}$ to achieve a higher signal-to-noise ratio for product ions, with a collision potential of between 20 and $50 \mathrm{~V}$ as required. The collision gas was argon and fragmentation was performed in the "transfer" cell (after ion mobility analysis), allowing product ion selection based on drift time and improving the MS/MS signal-to-noise ratio.

Open laser beam experiments were only carried out, and high-voltage interlocks were defeated, after consultation with 
H\&S staff at the University of Reading, and in accordance with the site-specific health and safety regulations.

Data Analysis. For spectral deconvolution, UniDec (version 1.0.10, University of Oxford, Oxford, UK) ${ }^{35}$ was used with the following parameters: $\mathrm{m} / z$ range, 600-2000; subtract curved, 1; Gaussian smoothing, 0; bin, 1.0; charge range, 1-50; mass range, 100-30000 Da; sample mass every $20 \mathrm{Da}$. Other parameters were unchanged from the default.

With Abstract Model Builder [Beta] (AMX, version 1.0.1563.0, prototype software developed by Waters), the data were automatically divided by the sample, detected by the change in TIC on a "burn" chromatogram (see Figure S3). Mass spectral peaks were binned to a window of $m / z 1$ (Figure S5). Background correction and normalization were applied. This meant that many isotopic peaks of highly charged ions were counted within the same bin. A narrower bin size was not beneficial to the model and required greater computation resources. The unsupervised PCA models were constructed from the first 10 dimensions and the supervised LDA model with 1 dimension for the $\mathrm{m} / z$ range of $600-2000$. The targeted model was built with a narrow $m / z$ range of $720-740$. Model cross-validation was performed using the built-in "leave $20 \%$ out" cross-validation with "standard deviation $>5$ " as the outlier definition.

\section{ASSOCIATED CONTENT}

\section{S Supporting Information}

The Supporting Information is available free of charge on the ACS Publications website at DOI: 10.1021/acsomega.9b01476.

Representative liquid AP-MALDI-TOF MS spectra for healthy and mastitis milk extracts acquired over $1 \mathrm{~min}$; AP-MALDI-IM-TOF MS spectrum of the $\mathrm{m} / \mathrm{z}$ range 500-1000 showing multiply charged ions in a mastitis sample; distinctive 'burn' chromatogram and mass spectrum of the selected region, produced by APMALDI-TOF MS and viewed in AMX; AP-MALDI CID MS/MS spectra for two larger peptides in a mastitis sample; and visualization of the binning applied to the mass spectra in AMX (PDF)

\section{AUTHOR INFORMATION}

\section{Corresponding Author}

*E-mail: r.k.cramer@reading.ac.uk.

\section{ORCID}

Rainer Cramer: 0000-0002-8037-2511

\section{Present Address}

"School of Biosciences, University of Birmingham, Edgbaston, Birmingham, B15 2TT, UK.

\section{Author Contributions}

O.J.H. performed the analyses and contributed to the design of the study. R.C. directed the project, conceived the study, and contributed to the analyses. O.J.H. and R.C. wrote the manuscript. M.M. contributed to the analyses and reviewed the manuscript. B.J. and C.R. provided the milk samples and metadata and reviewed the manuscript.

\section{Notes}

The authors declare no competing financial interest.

Abstract Model Builder (AMX) is available under beta license agreement on request from Waters Corporation. Requests should be addressed to Michael Morris (Waters Corporation).
Data supporting the results reported in this paper are openly available from the University of Reading Research Data Archive at http://dx.doi.org/10.17864/1947.169.

\section{ACKNOWLEDGMENTS}

This research was supported by the Engineering and Physical Sciences Research Council (EPSRC) (DTG grant no. 1498422) and Waters Corporation. Many thanks are extended to Emrys Jones (Waters) for support with the AMX software.

\section{REFERENCES}

(1) Whelan, L. C.; Power, K. A. R.; McDowell, D. T.; Kennedy, J.; Gallagher, W. M. Applications of SELDI-MS technology in oncology. J. Cell. Mol. Med. 2008, 12, 1535-1547.

(2) van Belkum, A.; Chatellier, S.; Girard, V.; Pincus, D.; Deol, P.; Dunne, W. M. Progress in proteomics for clinical microbiology: MALDI-TOF MS for microbial species identification and more. Expert Rev. Proteomics 2015, 12, 595-605.

(3) Cramer, R.; Pirkl, A.; Hillenkamp, F.; Dreisewerd, K. Liquid APUV-MALDI enables stable ion yields of multiply charged peptide and protein ions for sensitive analysis by mass spectrometry. Angew. Chem., Int. Ed. 2013, 52, 2364-2367.

(4) Dilillo, M.; de Graaf, E. L.; Yadav, A.; Belov, M. E.; McDonnell, L. A. Ultraviolet Photodissociation of ESI- and MALDI-Generated Protein Ions on a Q-Exactive Mass Spectrometer. J. Proteome Res. 2019, 18, 557-564.

(5) Zhurov, K. O.; Fornelli, L.; Wodrich, M. D.; Laskay, Ü. A.; Tsybin, Y. O. Principles of electron capture and transfer dissociation mass spectrometry applied to peptide and protein structure analysis. Chem. Soc. Rev. 2013, 42, 5014-5030.

(6) Ryumin, P.; Brown, J.; Morris, M.; Cramer, R. Protein identification using a nanoUHPLC-AP-MALDI MS/MS workflow with CID of multiply charged proteolytic peptides. Int. J. Mass Spectrom. 2017, 416, 20-28.

(7) Ruegg, P. L. A 100-Year Review: Mastitis detection, management, and prevention. J. Dairy Sci. 2017, 100, 10381-10397.

(8) Hogeveen, H.; Huijps, K.; Lam, T. Economic aspects of mastitis: new developments. N. Z. Vet. J. 2011, 59, 16-23.

(9) Verma, A.; Ambatipudi, K. Challenges and opportunities of bovine milk analysis by mass spectrometry. Clin. Proteomics 2016, 13, 8.

(10) Haug, A.; Høstmark, A. T.; Harstad, O. M. Bovine milk in human nutrition - a review. Lipids Health Dis. 2007, 6, 25.

(11) Hogarth, C. J.; Fitzpatrick, J. L.; Nolan, A. M.; Young, F. J.; Pitt, A.; Eckersall, P. D. Differential protein composition of bovine whey: a comparison of whey from healthy animals and from those with clinical mastitis. Proteomics 2004, 4, 2094-2100.

(12) Barnum, D. A.; Newbould, F. H. The Use of the California Mastitis Test for the Detection Of Bovine Mastitis. Can. Vet. J. 1961, 2, 83-90.

(13) Polat, B.; Colak, A.; Cengiz, M.; Yanmaz, L. E.; Oral, H.; Bastan, A.; Kaya, S.; Hayirli, A. Sensitivity and specificity of infrared thermography in detection of subclinical mastitis in dairy cows. $J$. Dairy Sci. 2010, 93, 3525-3532.

(14) Ferreira, J. C.; Gomes, M. S.; Bonsaglia, E. C. R.; Canisso, I. F.; Garrett, E. F.; Stewart, J. L.; Zhou, Z.; Lima, F. S. Comparative analysis of four commercial on-farm culture methods to identify bacteria associated with clinical mastitis in dairy cattle. PLoS One 2018, 13, No. e0194211.

(15) Viguier, C.; Arora, S.; Gilmartin, N.; Welbeck, K.; O’Kennedy, R. Mastitis detection: current trends and future perspectives. Trends Biotechnol. 2009, 27, 486-493.

(16) Sargeant, J. M.; Leslie, K. E.; Shirley, J. E.; Pulkrabek, B. J.; Lim, G. H. Sensitivity and Specificity of Somatic Cell Count and California Mastitis Test for Identifying Intramammary Infection in Early Lactation. J. Dairy Sci. 2001, 84, 2018-2024. 
(17) Albenzio, M.; Caroprese, M. Differential leucocyte count for ewe milk with low and high somatic cell count. J. Dairy Res. 2010, 78, $43-48$.

(18) Alonso-Fauste, I.; Andrés, M.; Iturralde, M.; Lampreave, F.; Gallart, J.; Álava, M. A. Proteomic characterization by 2-DE in bovine serum and whey from healthy and mastitis affected farm animals. $J$. Proteomics 2012, 75, 3015-3030.

(19) Baeker, R.; Haebel, S.; Schlatterer, K.; Schlatterer, B. Lipocalintype prostaglandin D synthase in milk: a new biomarker for bovine mastitis. Prostaglandins Other Lipid Mediators 2002, 67, 75-88.

(20) Magro, M.; Zaccarin, M.; Miotto, G.; Da Dalt, L.; Baratella, D.; Fariselli, P.; Gabai, G.; Vianello, F. Analysis of hard protein corona composition on selective iron oxide nanoparticles by MALDI-TOF mass spectrometry: identification and amplification of a hidden mastitis biomarker in milk proteome. Anal. Bioanal. Chem. 2018, 410, 2949-2959.

(21) Smolenski, G.; Haines, S.; Kwan, F. Y.-S.; Bond, J.; Farr, V.; Davis, S. R.; Stelwagen, K.; Wheeler, T. T. Characterisation of host defence proteins in milk using a proteomic approach. J. Proteome Res. 2007, 6, 207-215.

(22) Kusebauch, U.; Hernández-Castellano, L. E.; Bislev, S. L.; Moritz, R. L.; Røntved, C. M.; Bendixen, E. Selected reaction monitoring mass spectrometry of mastitis milk reveals pathogenspecific regulation of bovine host response proteins. J. Dairy Sci. 2018, $101,6532-6541$.

(23) Zoldan, K.; Schneider, J.; Moellmer, T.; Fueldner, C.; Knauer, J.; Fuerll, M.; Starke, A.; Specht, M.; Reiche, K.; Hackermueller, J.; Kalkhof, S.; von Bergen, M.; Bergfeld, U.; Fischer, R.; Pache, S.; Lehmann, J. Discovery and Validation of Immunological Biomarkers in Milk for Health Monitoring of Dairy Cows - Results from a Multiomics Approach. J. Adv. Dairy. Rep. 2017, 5, 1000182.

(24) St John, E. R.; Balog, J.; McKenzie, J. S.; Rossi, M.; Covington, A.; Muirhead, L.; Bodai, Z.; Rosini, F.; Speller, A. V. M.; Shousha, S.; Ramakrishnan, R.; Darzi, A.; Takats, Z.; Leff, D. R. Rapid evaporative ionisation mass spectrometry of electrosurgical vapours for the identification of breast pathology: towards an intelligent knife for breast cancer surgery. Breast Cancer Res. 2017, 19, 59.

(25) Woolman, M.; Ferry, I.; Kuzan-Fischer, C. M.; Wu, M.; Zou, J.; Kiyota, T.; Isik, S.; Dara, D.; Aman, A.; Das, S.; Taylor, M. D.; Rutka, J. T.; Ginsberg, H. J.; Zarrine-Afsar, A. Rapid determination of medulloblastoma subgroup affiliation with mass spectrometry using a handheld picosecond infrared laser desorption probe. Chem. Sci. 2017, $8,6508-6519$.

(26) Kostrzewa, M. Application of the MALDI Biotyper to clinical microbiology: progress and potential. Expert Rev. Proteomics 2018, 15, 193-202.

(27) Cramer, R.; Corless, S. The nature of collision-induced dissociation processes of doubly protonated peptides: comparative study for the future use of matrix-assisted laser desorption/ionization on a hybrid quadrupole time-of-flight mass spectrometer in proteomics. Rapid Commun. Mass Spectrom. 2001, 15, 2058-2066.

(28) Meltretter, J.; Schmidt, A.; Humeny, A.; Becker, C.-M.; Pischetsrieder, M. Analysis of the peptide profile of milk and its changes during thermal treatment and storage. J. Agric. Food Chem. 2008, 56, 2899-2906.

(29) Baum, F.; Fedorova, M.; Ebner, J.; Hoffmann, R.; Pischetsrieder, M. Analysis of the endogenous peptide profile of milk: identification of 248 mainly casein-derived peptides. J. Proteome Res. 2013, 12, 5447-5462.

(30) Lahov, E.; Regelson, W. Antibacterial and immunostimulating casein-derived substances from milk: Casecidin, isracidin peptides. Food Chem. Toxicol. 1996, 34, 131-145.

(31) Pyörälä, S. Indicators of inflammation in the diagnosis of mastitis. Vet. Res. 2003, 34, 565-578.

(32) Guerreiro, T. M.; de Oliveira, D. N.; Melo, C. F. O. R.; de Oliveira Lima, E.; Ribeiro, M. D. S.; Catharino, R. R. Evaluating the effects of the adulterants in milk using direct-infusion high-resolution mass spectrometry. Food Res. Int. 2018, 108, 498-504.
(33) Ryumin, P.; Cramer, R. The composition of liquid atmospheric pressure matrix-assisted laser desorption/ionization matrices and its effect on ionization in mass spectrometry. Anal. Chim. Acta 2018, 1013, 43-53.

(34) Ryumin, P.; Brown, J.; Morris, M.; Cramer, R. Investigation and optimization of parameters affecting the multiply charged ion yield in AP-MALDI MS. Methods 2016, 104, 11-20.

(35) Marty, M. T.; Baldwin, A. J.; Marklund, E. G.; Hochberg, G. K. A.; Benesch, J. L. P.; Robinson, C. V. Bayesian Deconvolution of Mass and Ion Mobility Spectra: From Binary Interactions to Polydisperse Ensembles. Anal. Chem. 2015, 87, 4370-4376. 